\title{
Domain wall dynamics in expanding spaces
}

\author{
Francisco J. Cao, ${ }^{1,2, *}$ Elías Zamora-Sillero, ${ }^{3, \dagger}$ and Niurka R. Quintero ${ }^{3, \$}$ \\ ${ }^{1}$ Departamento de Física Atómica, Molecular y Nuclear, Universidad Complutense de Madrid, \\ Avenida Complutense s/n, E-28040 Madrid, Spain \\ ${ }^{2}$ LERMA, Observatoire de Paris, Laboratoire Associé au CNRS UMR 8112, 61 Avenue de l'Observatoire, 75014 Paris, France \\ ${ }^{3}$ Departamento de Física Aplicada I, E.U.P., Universidad de Sevilla, Virgen de África 7, 41011 Sevilla, Spain
}

(Received 1 December 2005; published 12 May 2006)

\begin{abstract}
We study the effects on the dynamics of kinks due to expansions and contractions of the space. We show that the propagation velocity of the kink can be adiabatically tuned through slow expansions and/or contractions, while its width is given as a function of the velocity. We also analyze the case of fast expansions and/or contractions, where we are no longer on the adiabatic regime. In this case the kink moves more slowly after an expansion-contraction cycle as a consequence of the loss of energy through radiation. All these effects are numerically studied in the nonlinear Klein-Gordon equations (both for the sine-Gordon and for the $\phi^{4}$ potential), and they are also studied within the framework of the collective coordinate evolution equations for the width and the center of mass of the kink. These collective coordinate evolution equations are obtained with a procedure that allows us to consider even the case of large expansions and/or contractions.
\end{abstract}

DOI: 10.1103/PhysRevE.73.056603

PACS number(s): 05.45.Yv, 45.20.Jj, 02.30.Jr

\section{INTRODUCTION}

Domain walls are the interface between two regions where the system is close to two different stable or metastable states. Their formation and dynamics are relevant for many domains of physics as solid state physics (magnetic materials) [1,2], liquid crystals science [3], plasma physics $[4,5]$, and cosmology (phase transitions in the early universe) $[6,7]$. Spatially extended systems with more than one stable or metastable state can present domain walls, as is the case of systems that have undergone a first order phase transition. An expansion or contraction of these systems can be realized through an external action on the system (magnetic materials, liquid crystals, plasmas), or they are already present in the system dynamics (phase transitions in the early universe).

The kink solutions of the one-dimensional nonlinear Klein-Gordon equation for potentials with two or more stable states (as sine-Gordon or $\varphi^{4}$ ) provide models for the propagation of plane domain walls in the direction perpendicular to the domain wall. These models have been successfully used in several contexts as in the description of the dynamics of domain walls in ferrodistortive materials [2], and the magnetohydrodynamic mode trajectory in reversedfield pinch experiments [4,5]. On the other hand, expansions and contractions can be realized in magnetic materials through the mechanical action on the material [8], and in plasmas through confinement [9]; while in the case of phase transitions in the early universe, the expansion is already present in the system dynamics. These expansions and/or contractions can be parametrized with a scale factor $a(t)$ when they are homogeneous in space [10]. The characteristic times for the time variation of $a(t)$ can be shorter or longer than the characteristic times of the kink dynamic.

\footnotetext{
*Electronic address: francao@fis.ucm.es

†Electronic address: elias@euler.us.es

‡Electronic address: niurka@euler.us.es
}

With these facts in mind, in this paper we consider kinks of the nonlinear Klein-Gordon equations for the sine-Gordon and the $\phi^{4}$ potentials (as a paradigmatic examples of integrable and nonintegrable nonlinear Klein-Gordon equations, respectively), and we study their dynamics in slow and fast expanding and/or contracting spaces.

In order to achieve this purpose, our paper is organized as follows: In Sec. II we introduce the nonlinear Klein-Gordon equations in expanding and/or contracting spaces, and derive the continuity equations for the energy and momentum densities. In the next section, Sec. III, we use the Rice Ansatz [11] in these continuity equations, and we reduce the problem with an infinite number of degrees of freedom to an approximate description in terms of two collective coordinates (CC), the center and the width of the kink. In Sec. IV, we study the kink dynamic in both slow and fast expanding and/or contracting spaces using the collective coordinate evolution equations, and integrating numerically the complete nonlinear Klein-Gordon equations. We first consider the case of the slow expansion and/or contraction within the adiabatic approximation, and later we discuss the fast expansion and/or contraction case and the departures from the adiabaticity that emerge. Finally, in the conclusions, we summarize and discuss the results.

The previous sections are complemented with two Appendixes where some technical details related to expanding and/or contracting spaces are reviewed, and the equivalence between several collective coordinates approaches [12-14] to this problem is shown.

\section{KLEIN-GORDON EQUATION IN EXPANDING SPACES}

The Klein-Gordon equation in nonexpanding spaces can be obtained by extremizing the action defined by the Lagrangian density,

$$
S=\int d t d x \mathcal{L}=\int d t d x\left[\frac{1}{2} \phi_{t}^{2}-\frac{1}{2} \phi_{x}^{2}-U(\phi)\right] .
$$


In an expanding (or contracting) space with one spatial dimension the space-time distance between events is given by

$$
d s^{2}=d t^{2}-a^{2}(t) d x^{2}
$$

where $a(t)$ is the scale factor that gives the dilation of the physical spatial distances (see Appendix A). Thus, the physical spatial distance between two points with coordinates $x_{1}$ and $x_{2}$ is

$$
d_{12}(t)=a(t)\left|x_{2}-x_{1}\right| .
$$

The Klein-Gordon equation in this expanding space can be obtained noting that after the spatial dilation $d x \rightarrow a(t) d x$ the action for a Klein-Gordon field is

$$
S=\int d t d x a(t) \mathcal{L}=\int d t d x a(t)\left[\frac{1}{2} \phi_{t}^{2}-\frac{1}{2} \frac{\phi_{x}^{2}}{a^{2}(t)}-U(\phi)\right],
$$

where the subscripts $t$ and $x$ indicate the time and space partial derivatives, respectively, and $U(\phi)$ is the nonlinear Klein-Gordon potential.

The Euler-Lagrange equation for the field $\phi$,

$$
\frac{\partial}{\partial t} \frac{\delta[a(t) \mathcal{L}]}{\delta \phi_{t}}+\frac{\partial}{\partial x} \frac{\delta[a(t) \mathcal{L}]}{\delta \phi_{x}}-\frac{\delta[a(t) \mathcal{L}]}{\delta \phi}=0,
$$

gives the equation of motion

$$
\phi_{t t}+H(t) \phi_{t}-\frac{\phi_{x x}}{a^{2}(t)}+\frac{d U}{d \phi}=0
$$

with $H=a_{t} / a$.

We have to keep in mind that the physical distance is $a(t)\left|x_{2}-x_{1}\right|$ and not $\left|x_{2}-x_{1}\right|$ (the latter is usually called the comoving distance). Thus, when $a(t)$ grows the space experiences an elongation and when $a(t)$ decreases the space experiences a contraction.

We define the energy density $\rho_{E}(t, x)$, the momentum density $\rho_{P}(t, x)$, and the momentum current $j_{P}(t, x)$, as the timetime, space-time, and space-space contravariant components, respectively, of the energy-momentum tensor. We obtain

$$
\begin{gathered}
\rho_{E}=\frac{1}{2} \phi_{t}^{2}+\frac{1}{2} \frac{\phi_{x}^{2}}{a^{2}}+U(\phi), \\
\rho_{P}=-\frac{1}{a^{2}} \phi_{t} \phi_{x}, \\
j_{P}=\frac{1}{a^{2}}\left(\frac{1}{2} \phi_{t}^{2}+\frac{1}{2} \frac{\phi_{x}^{2}}{a^{2}}-U(\phi)\right) .
\end{gathered}
$$

The energy-momentum conservation (in the covariant sense) gives the following relations:

$$
\frac{\partial \rho_{E}}{\partial t}+H\left(\rho_{E}+a^{2} j_{P}\right)+\frac{\partial \rho_{P}}{\partial x}=0
$$

$$
\frac{\partial \rho_{P}}{\partial t}+3 H \rho_{P}+\frac{\partial j_{P}}{\partial x}=0
$$

that also can be obtained using the equation of motion (6).

This also implies relations between the total energy $E$ $=\int a d x \rho_{E}$, the total momentum $P=\int a d x \rho_{P}$, and the total momentum current $J_{P}=\int a d x j_{P}$. They are given by

$$
\frac{d E}{d t}+H a^{2} J_{P}=0
$$

$\left[\right.$ provided $\left.\rho_{P}(t, \infty)=\rho_{P}(t,-\infty)\right]$, and

$$
\frac{d P}{d t}+2 H P=0
$$

[provided $\left.j_{P}(t, \infty)=j_{P}(t,-\infty)\right]$. Note that for a space that is neither expanding nor contracting [i.e., $a(t)=1] E$ and $P$ are conserved quantities [1].

\section{COLLECTIVE COORDINATES ANALYSIS FOR THE PERTURBED KLEIN-GORDON EQUATION}

In the previous section we have shown that the momentum and the energy are no longer conserved quantities in expanding and/or contracting spaces, instead they satisfy the continuity equations (12) and (13), respectively. We are interested in the evolution of the kink which we describe through two collective coordinates, its width and its center of mass position. Therefore, we neglect the possible energy transfer to other degrees of freedom.

In order to obtain the equations of motion for these two CCs we use the Rice Ansatz [11] in the continuity equations (12) and (13) [with the energy and momentum given by Eqs. (7)-(9)], and we study two particular nonlinear potentials, the sine-Gordon (SG) potential $U(\phi)=1-\cos (\phi)$, and the $\phi^{4}$ potential $U(\phi)=\frac{1}{4}\left(1-\phi^{2}\right)^{2}$. The domain walls in these systems are represented by kinklike solutions that describe the transition between two regions in different minima of the potential. For the sine-Gordon potential the Rice Ansatz assumes the function

$$
\phi(x, t)=4 \arctan \left\{\exp \left[\frac{x-X(t)}{l(t)}\right]\right\},
$$

whereas for the $\phi^{4}$, the Rice Ansatz reads

$$
\phi(x, t)=\tanh \left[\frac{x-X(t)}{l(t)}\right]
$$

where $X(t)$ and $l(t)$ represent in both cases the center and the width of the kink, respectively, in the co-moving frame. Note that for the unperturbed system [take, in Eq. (6), $a(t)=1]$ the kinklike solutions are represented by Eqs. (14) and (15) with $X(t)=v t$ and $l(t)=l_{s}=l_{0} \sqrt{1-v^{2}}$, being $v$ and $l_{s}$ the constant velocity and width of the kink, respectively, and $l_{0}=1\left(l_{0}\right.$ $=\sqrt{2})$ the width of the soliton at rest for SG $\left(\varphi^{4}\right)$. The time dependence of the scale factor $a(t)$ (expansions and/or contractions) implies changes in the width of the kink, as we show later, justifying the choice of the Rice Ansatz.

Inserting the Ansatz, Eq. (14) or (15), in the expressions 
for $\rho_{P}, \rho_{E}$, and $j_{P}$ and integrating over $x$, we obtain

$$
\begin{gathered}
P(t)=\frac{M_{0} l_{0} \dot{X}}{a(t) l}, \\
E(t)=\frac{M_{0} l_{0}}{2} \frac{a \dot{X}^{2}}{l}+\frac{\alpha M_{0} l_{0}}{2} \frac{a \dot{l}^{2}}{l}+\frac{M_{0}}{2}\left(\frac{l_{0}}{a l}+\frac{a l}{l_{0}}\right), \\
J_{P}(t)=\frac{M_{0} l_{0}}{2} \frac{\dot{X}^{2}}{a l}+\frac{\alpha M_{0} l_{0}}{2} \frac{\dot{l}^{2}}{a l}+\frac{M_{0}}{2 a^{2}}\left(\frac{l_{0}}{a l}-\frac{a l}{l_{0}}\right),
\end{gathered}
$$

respectively; where $\alpha=\pi^{2} / 12$ and $M_{0}=8$ for the sineGordon and $\alpha=\left(\pi^{2}-6\right) / 12$ and $M_{0}=2 \sqrt{2} / 3$ for $\varphi^{4}$. These expressions are replaced in the continuity equations (12) and (13), giving the following systems of ordinary differential equations (ODE) for $X(t)$ and $l(t)$ :

$$
\begin{gathered}
\dot{X}=\frac{a(t) P(t) l(t)}{M_{0} l_{0}}, \\
\frac{d P}{d t}=-2 H P, \\
\alpha\left[\dot{l}^{2}-2 H l \dot{l}-2 \ddot{l}\right]=\frac{l^{2}}{l_{0}^{2}}\left(1+a^{2}(t) \frac{P^{2}}{M_{0}^{2}}\right)-\frac{1}{a^{2}},
\end{gathered}
$$

where the dots denote the derivative with respect to $t$. If the initial conditions are those of a kink with an initial position $X(0)$ and an initial velocity $\dot{X}(0)$, this implies $l(0)=l_{0} \sqrt{1-\dot{X}^{2}(0)}$ and $\dot{l}(0)=0$, and $P(0)$ is determined by Eq. (19) at $t=0$. The same collective coordinates evolution equations are obtained using the Lagrangian method $[14,15]$ or the generalized travelling wave Ansatz (GTWA) $[12,16]$ (based on projection techniques), as it is shown in Appendix B.

This set of evolution equations, Eqs. (19)-(21), involves the variables $X(t)$ and $l(t)$ through the momentum $P(t)$ defined in Sec. II. Note that the equation for the momentum is linear, and therefore it can be solved exactly yielding

$$
P(t)=\frac{P(0)}{a^{2}(t)},
$$

with $P(0)=\dot{X}(0) M_{0} l_{0} / \sqrt{1-\dot{X}^{2}(0)}$. The other two equations are nonlinear and coupled, and they are analyzed in detail in the next section.

\section{KINK DYNAMICS IN EXPANDING AND/OR CONTRACTING SPACES}

First of all, let us remark that the main physical variables associated with the kink propagation are the physical center of the kink, $X_{\text {phys }}(t)=a(t) X(t)$, its physical width $l_{\text {phys }}(t)$ $=a(t) l(t)$, and its physical velocity

$$
V_{\text {phys }}(t)=H X_{\text {phys }}(t)+a(t) \frac{d X(t)}{d t} \text {. }
$$

The first term on the rhs is the contribution to the kink velocity due to the expansion or contraction of the space (i.e., it turns to zero when the space finishes its elongation or contraction $H=0$ ). On the other hand, the second term is the peculiar velocity of the kink with respect to the propagating space,

$$
V_{p e c}(t)=a(t) \frac{d X(t)}{d t}
$$

For the sake of clarity, we analyze separately the cases of slow and fast expansion and/or contraction.

\section{A. Slow expansion and/or contraction (adiabatic regime)}

Note from Eqs. (19)-(21) that the adiabatic approximation implies

$$
\alpha\left[\dot{l}^{2}-2 H l i-2 \ddot{l}\right] \ll \frac{1}{a^{2}} .
$$

Hence, from Eq. (21) we obtain the relation

$$
l_{\text {phys }}(t)=l_{0} \sqrt{1-V_{p e c}^{2}(t)}
$$

that links the physical width and the peculiar velocity of the kink. Furthermore, from Eqs. (19) and (22)-(26) we obtain

$$
V_{p e c}(t)=\frac{P(0)}{\sqrt{M_{0}^{2} a^{2}(t)+P^{2}(0)}} .
$$

From the previous Eqs. (26) and (27) we see that an expansion decelerates the peculiar motion of the kink and makes it wider, asymptotically

$$
\lim _{a(t) \rightarrow \infty} V_{p e c}(t)=0, \quad \lim _{a(t) \rightarrow \infty} l_{p h y s}(t)=l_{0} .
$$

On the other hand, a contraction accelerates the peculiar motion of the kink and makes it sharper, asymptotically

$$
\lim _{a(t) \rightarrow 0} V_{p e c}(t)=1, \quad \lim _{a(t) \rightarrow 0} l_{p h y s}(t)=0 .
$$

The adiabaticity conditions can be obtained from Eq. (25) and require only slow expansions and/or contractions

$$
\begin{gathered}
|H| \ll \frac{\Omega_{R}}{1-V_{p e c}^{2}}, \\
|\dot{H}| \ll \frac{\Omega_{R}^{2}}{\left(1-V_{p e c}^{2}\right)^{2}},
\end{gathered}
$$

where $\Omega_{R}=1 /\left(\sqrt{\alpha} l_{0}\right)$ is the so-called Rice frequency for zero velocity, $\Omega_{R}=\sqrt{12} / \pi=1.10 \ldots$, for $\mathrm{SG}$ and $\Omega_{R}=\sqrt{6 /\left(\pi^{2}-6\right)}$ $=1.24 \ldots$, for $\varphi^{4}$.

We have studied the dynamics for different expansion and contraction rates, both for the sine-Gordon and the $\phi^{4}$ potentials. In particular, Fig. 1 (and also Fig. 2) shows the results 

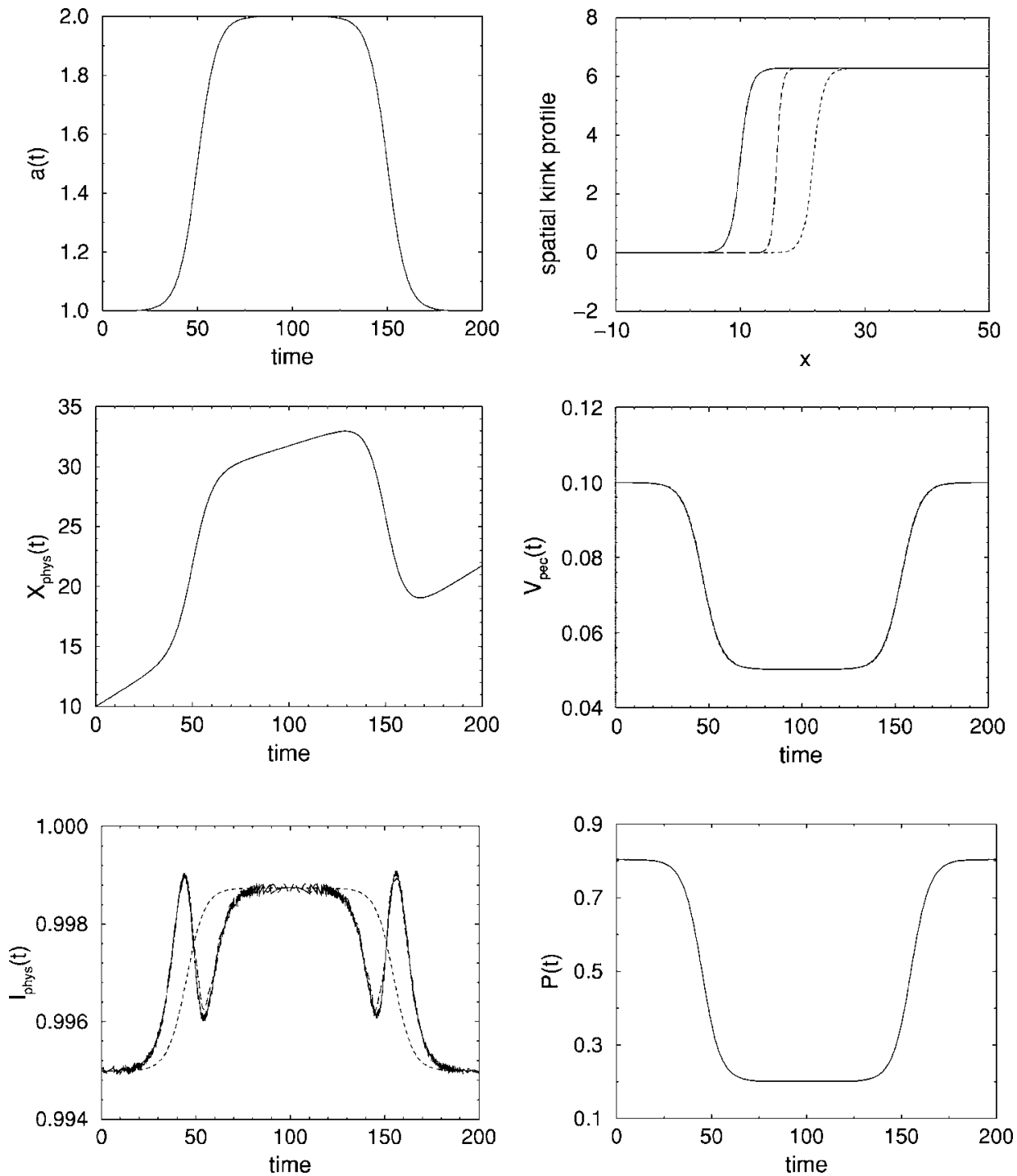

FIG. 1. Sine-Gordon kink dynamics in a slow expanding and later contracting space. Top-left panel: scale factor $a(t)$, Eq. (32) with $\Delta a=1, t_{0}=50, t_{1}=150$, and $\Delta t=10$. Top-right panel: spatial profile of the kink $\phi\left(x, t_{f i x}\right)$ at times $t_{f i x}=0$ (solid line), $t_{f i x}=100$ (long-dashed line), and $t_{f i x}=200$ (dashed line). Three following panels: position of the center of the kink $X_{\text {phys }}(t)$, peculiar kink velocity $V_{p e c}(t)$, and width of the kink $l_{\text {phys }}(t)$ as functions of time given by the numerical simulations of the partial differential equation [PDE] (solid line), the numerical solutions of the CCs equation (long-dashed line), and the adiabatic approximation (dashed line). Bottom-right panel: momentum of the kink $P(t)$ exact solution. Initial conditions: a kink with $X(0)=10$ and $\dot{X}(0)=0.1$. for an expansion followed by a contraction parametrized by the function

$$
a(t)=1+\frac{\Delta a}{2} \tanh \left(\frac{t-t_{0}}{\Delta t}\right)-\frac{\Delta a}{2} \tanh \left(\frac{t-t_{1}}{\Delta t}\right),
$$

with $\Delta a, t_{0}, t_{1}$, and $\Delta t$ constants. ( $\Delta a$ represents the changes in $a ; t_{0}$ and $t_{1}$, the times when the expansion and contraction take place, respectively, and $\Delta t$ is the characteristic time interval where the changes in $a$ take place.)

In Fig. 1 we show the effects on a kink of a slow expansion followed by a slow contraction that verify the adiabatic conditions $\left[H \sim \Delta a / \Delta t=0.1\right.$ and $\left.\dot{H} \sim \Delta a /(\Delta t)^{2}=0.01\right]$. In this case, when the adiabatic conditions are verified, both the CCs equations and the adiabatic approximations are in good agreement with the exact results, especially for the center of the kink $X_{\text {phys }}(t)$ and its peculiar velocity $V_{p e c}(t)$ (see Fig. 1). It is important to note that the agreement is good even when the variations of $a$ are large.

This implies that the main effects of a slow expansion and/or contraction are the change of the width and the speed of the propagation of the kink, following the adiabatic rela- tions (26) and (27). Therefore, these results show that the speed of a kink can be tuned by slowly expanding and/or contracting the space.

Note that the CCs evolution equations go further and are able to predict the small oscillations of the kink width [23] produced by the slow expansion and/or contraction (see Fig. 1). Therefore, they can compute deviations from adiabaticity excluding the radiative effects (because they involve the transfer of energy to other degrees of freedom).

It is important to stress that the collective coordinate evolution equations obtained with an Ansatz with fixed width and a variable center of the kink does not predict any variation of the kink speed due to expansions and/or contractions. Therefore, an Ansatz that allows the width of the kink to evolve, like the Rice Ansatz, is an essential ingredient in order to obtain the correct variation of the speed of the kink under expansions and/or contractions. It is interesting to remark that, in the $\phi^{4}$ model, the oscillations of the width are related to excitations of the internal mode [11], while in the sine-Gordon equation it has been shown that the excitation of certain phonons can imply oscillations in the shape of the kink $[17,18]$. 

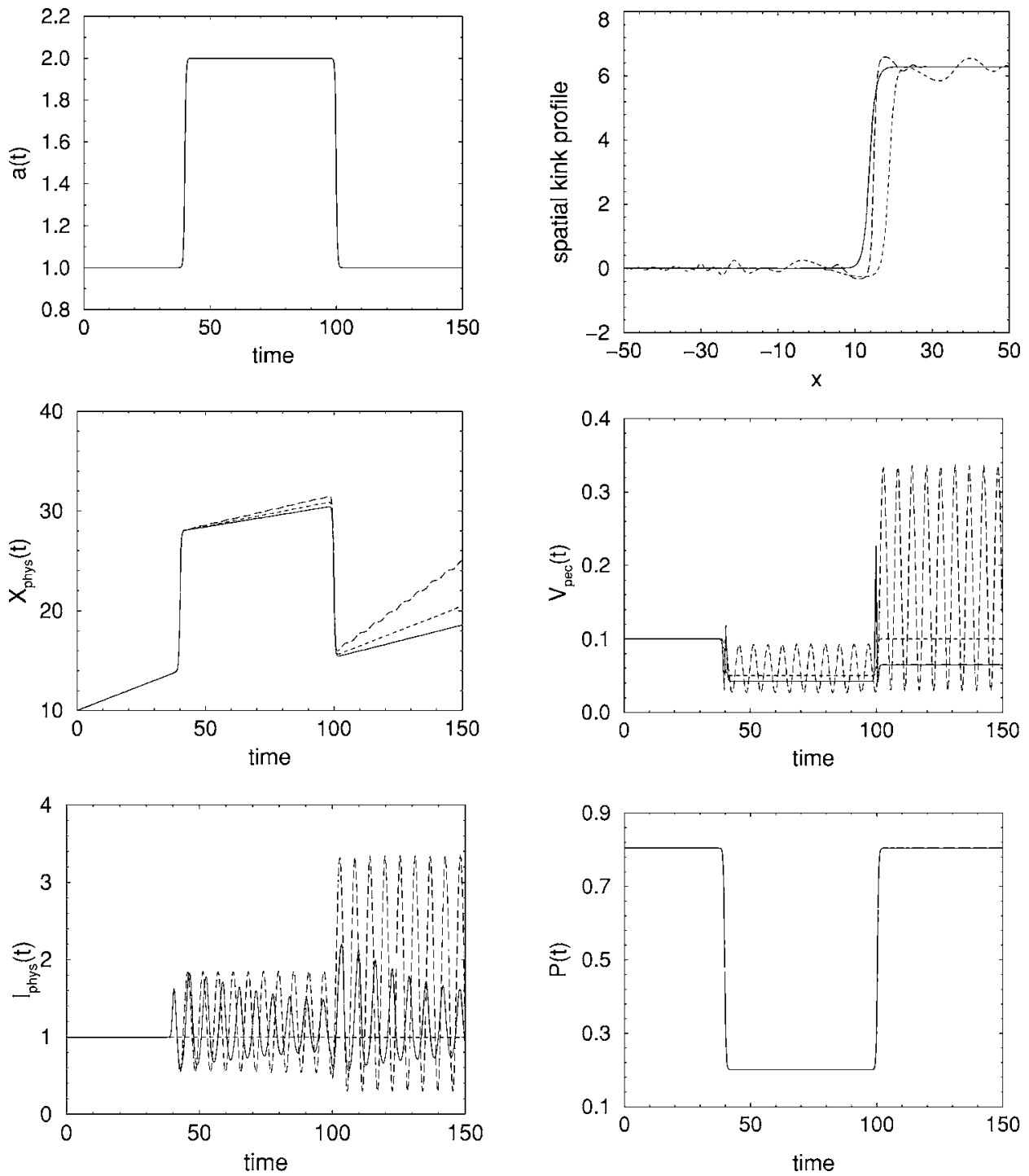

FIG. 2. Sine-Gordon kink dynamics in a fast expanding and later contracting space. Top-left panel: scale factor $a(t)$, Eq. (32) with $\Delta a=1, t_{0}=40, t_{1}=100$ and $\Delta t=0.5$. Top-right panel: spatial profile of the kink $\phi\left(x, t_{f i x}\right)$ at times $t_{f i x}=37.5$ (solid line), $t_{f i x}$ $=75$ (long-dashed line), and $t_{f i x}$ $=150$ (dashed line). The three following panels: position of the center of the kink $X_{\text {phys }}(t)$, peculiar kink velocity $V_{p e c}(t)$, and width of the kink $l_{\text {phys }}(t)$ as functions of time given by the numerical simulations of the PDE (solid line), the numerical solutions of the CCs equation (long-dashed line), and the adiabatic approximation (dashed line). Bottom-right panel: momentum of the kink $P(t)$ exact solution. Initial conditions: a kink with $X(0)=10$ and $\dot{X}(0)=0.1$.
On the other hand, in general, the applicability of the perturbative approaches is restricted to slow and small expansions and/or contractions. The perturbative approaches rewrite Eq. (6) in the form $\phi_{t t}-\phi_{x x}+d U / d \phi=\epsilon f \equiv-H(t) \phi_{t}$ $-\left[1 / a^{2}(t)-1\right] \phi_{x x}$, where $\epsilon f$ is treated as a small perturbation. Therefore, their range of applicability is limited not only to small $H$ but also to small $\left(1 / a^{2}-1\right)$, i.e., slow and small expansions and/or contractions.

\section{B. Fast expansion and/or contraction (nonadiabatic regime)}

When the expansion and/or contraction is faster the adiabatic approximation breaks, and nonadiabatic effects appear as radiation (compare the kink profiles in Figs. 1 and 2) and as a change in the final width and velocity of the kink [that are no longer those predicted by Eqs. (26) and (27)]. (See Fig. 2, that corresponds to the nonadiabatic regime $H \sim \Delta a / \Delta t=2$ and $\dot{H} \sim \Delta a /(\Delta t)^{2}=4$.) The CCs evolution equations predict strong oscillations in the velocity and the width of the kink. In the numerical integration of the complete evolution equations [Eq. (6)] the oscillations are indeed present in the width. However, there are other degrees of freedom to which the energy can be transferred. This results in the damping of the oscillations through radiation emission. The loss of energy can also be easily shown noting that after an expansion-contraction cycle the kink moves slower (see Fig. 2).

We would like to stress that all the previous comments about slow and fast expansions and/or contractions apply both for the sine-Gordon and the $\phi^{4}$ potential. We have performed numerical simulations and comparisons of the adiabatic approximation, the CCs evolution equations, and the full evolution equation for both potentials obtaining analogous results.

\section{CONCLUSIONS}

We have studied the effects on a kink of slow and fast expansions and/or contractions of the media, both for the sine-Gordon and for the $\phi^{4}$ equations. We have used the Rice Ansatz [11] in the continuity equations for the momentum and the energy, in order to obtain the evolution equations for the width and the center of a kink in an expanding and/or contracting space. In general, this set of ODEs cannot be 
solved analytically and the solution must be obtained numerically. However, this effective description in terms of the collective coordinates usually gives relevant information and insight of the evolution and the phenomena involved [16,19-22]. In this case the CCs approximation is able to describe very accurately the dynamics of the kink when the process is slow, even when the variations of $a$ are large. We have found that the main effects of a slow expansion and/or contraction are the change of the width and the velocity of the kink, following the adiabatic relations (26) and (27). In addition, the collective coordinate evolution equations for the width and the center of the kink are able to take into account the small deviations from adiabaticity (excluding explicitly the radiative effects, because they are related to transference of energy to other degrees of freedom).

An important consequence of the adiabatic relations is that the speed of a kink can be tuned by a slowly expansion and/or contraction of space. Thus, this result provides a mechanism to control the speed of a kink whenever we can expand and contract the media where it propagates.

On the other hand, fast expansions and/or contractions break the adiabatic approximation, giving rise to nonadiabatic effects, as, for example, radiation, that imply a change in the final width and velocity of the kink (i.e., they are no longer those predicted by the adiabatic relations). The collective coordinate evolution equations predict strong oscillations in the width of the kink. In the full problem, these oscillations are also present. However, there are other degrees of freedom to which the energy of these oscillations can be transferred. This results in emission of radiation and in the damping of these oscillations. In this sense, the fast expansion and/or contraction of space provides a method to implement a fast change in the speed of the kink, and also to generate phonons.

\section{ACKNOWLEDGMENTS}

We acknowledge financial support from the Ministerio de Ciencia y Tecnología of Spain under Grants No. BFM200302547/FISI (F.J.C.), No. NAN2004-09087-C0303 (F.J.C.), and No. FIS2005-973 (E.Z.S., N.R.Q.), and from the Junta de Andalucía through the projects FQM-0207 and 00481 (E.Z.S., N.R.Q.). In addition, E.Z.S. thanks the University of Sevilla for its financial support.

\section{APPENDIX A: EXPANDING SPACES}

This appendix includes some technical details related to expanding spaces.

\section{Metric}

The metric in an homogeneous and isotropous flat space is given by

$$
d s^{2}=d t^{2}-a^{2}(t) d x^{2}=g_{\mu \nu} d x^{\mu} d x^{\nu},
$$

where $a(t)$ is the scale factor for the expansion and/or contraction, the metric tensor is

$$
g_{\mu \nu}=\left(\begin{array}{cc}
1 & 0 \\
0 & -a^{2}(t)
\end{array}\right) .
$$

Its inverse, $g^{\mu \nu}$, defined by $g_{\mu \nu} g^{\nu \lambda}=g_{\mu}^{\lambda}=\delta_{\mu}^{\lambda}$ (with $\delta_{\mu}^{\lambda}$ a Kronecker delta) is

$$
g^{\mu \nu}=\left(\begin{array}{cc}
1 & 0 \\
0 & -\frac{1}{a^{2}(t)}
\end{array}\right) .
$$

The contraction with $g_{\mu \nu}$ lowers indices, and the contraction with $g^{\mu \nu}$ raises indices.

\section{Invariant element of space-time volume}

Defining

$$
g \equiv-\operatorname{Det} g_{\mu \nu}
$$

it can be shown ([10] pages 98 and 99) that

$$
\sqrt{g} d t d x
$$

is an invariant volume element under general coordinate transformations. ( $d t d x$ transforms with the Jacobian, while $\sqrt{g}$ transforms with the inverse of the Jacobian.)

Therefore the appropriate relation between the action $S$, and the Lagrangian density $\mathcal{L}$ is

$$
S=\int d t d x \sqrt{g} \mathcal{L} .
$$

This definition implies that if the action is invariant under general coordinate transformations the Lagrangian density is also invariant. In our case $\sqrt{g}=a(t)$.

\section{Energy-momentum tensor}

The energy-momentum tensor is

$$
T^{\mu \nu}=\partial^{\mu} \phi \partial^{\nu} \phi-g^{\mu \nu} \mathcal{L} .
$$

Some useful relations to calculate its components are

$$
\begin{gathered}
\partial^{x} \phi=g^{x \sigma} \partial_{\sigma} \phi=g^{x x} \partial_{x} \phi=-\frac{\phi_{x}}{a^{2}}, \\
\partial^{t} \phi=\partial_{t} \phi=\phi_{t} .
\end{gathered}
$$

The time-time component of the energy-momentum tensor or energy density is

$$
\begin{aligned}
\rho_{E} \equiv T^{t t} & =\partial^{t} \phi \partial^{t} \phi-g^{t t}\left[\frac{1}{2} \phi_{t}^{2}-\frac{1}{2} \frac{\phi_{x}^{2}}{a^{2}}-U(\phi)\right] \\
& =\frac{1}{2} \phi_{t}^{2}+\frac{1}{2} \frac{\phi_{x}^{2}}{a^{2}}+U(\phi),
\end{aligned}
$$

the space-time component or momentum density is

$$
\rho_{P} \equiv T^{x t}=T^{t x}=\partial^{t} \phi \partial^{x} \phi-g^{t x} \mathcal{L}=-\frac{1}{a^{2}} \phi_{t} \phi_{x},
$$

and the space-space component or momentum current is 


$$
j_{P} \equiv T^{x x}=\frac{1}{a^{2}}\left(\frac{1}{2} \phi_{t}^{2}+\frac{1}{2} \frac{\phi_{x}^{2}}{a^{2}}-U(\phi)\right) .
$$

\section{APPENDIX B: EQUIVALENCE AMONG DIFFERENT CC APPROACHES}

In this appendix we show that the evolution equations for the collective coordinates obtained with the derivation of the momentum and the energy [13] (that we used in Sec. III) are the same as those obtained with the Lagrangian method $[14,15]$ and with the so-called GTWA $[12,16]$ (based on projection techniques). In all cases, we use the Rice Ansatz as an approximated kinklike solution of the following perturbed nonlinear Klein-Gordon equation:

$$
\phi_{t t}-\frac{\phi_{x x}}{a^{2}(t)}=-\frac{d U}{d \phi}-[H(t)+\beta(t)] \phi_{t}+z(x, t, \phi),
$$

where $\beta(t)$ is a time-dependent damping coefficient and $z(x, t, \phi)$ represents a generic perturbation on the system. Note that for $\beta=0$ and $z(x, t, \phi)=0$ we recover the system (6), introduced in Sec. II.

\section{Generalized Lagrangian formalism}

Introducing a new time variable

$$
\tau \equiv \int_{0}^{t} \frac{d t^{\prime}}{c\left(t^{\prime}\right)}
$$

where $c(t)$ is given by

$c(t) \equiv \exp \left\{\int_{0}^{t}\left[H\left(t^{\prime}\right)+\beta\left(t^{\prime}\right)\right] d t^{\prime}\right\}=\frac{a(t)}{a(0)} \exp \left\{\int_{0}^{t} \beta\left(t^{\prime}\right) d t^{\prime}\right\}$,

Eq. (B1) becomes the dissipationless equation,

$$
\frac{\phi_{\tau \tau}}{C^{2}(\tau)}-\frac{\phi_{x x}}{A^{2}(\tau)}=-\frac{d U}{d \phi}+Z(x, \tau, \phi),
$$

with $C(\tau)=c[t(\tau)], A(\tau)=a[t(\tau)]$, and $Z(x, \tau, \phi)=z[x, t(\tau)$, $\phi]$.

The evolution equation (B4) can be obtained from the action

$$
\begin{aligned}
S=\int d \tau d x \mathcal{L}= & \int d \tau d x\left\{\frac{1}{2} \phi_{\tau}^{2}-\frac{1}{2} \frac{C^{2}(\tau)}{A^{2}(\tau)} \phi_{x}^{2}\right. \\
& \left.-C^{2}(\tau)\left[U(\phi)+M\left(x, \tau, \phi, \phi_{x}\right)\right]\right\}
\end{aligned}
$$

$\mathcal{L}$ being the Lagrangian density, and $M\left(x, \tau, \phi, \phi_{x}\right)$ an "Euler-Lagrange integral" of $Z(x, \tau, \phi)$. Some of the possible integrations are

(i) the $\phi$ functional integral

$$
M(x, \tau, \phi)=-\int \mathcal{D} \phi Z(x, \tau, \phi),
$$

and (ii) the following integration for the particular case where $Z(x, \tau, \phi)=F(x, \tau) G(\phi)[15]$ :

$$
M\left(x, \tau, \phi, \phi_{x}\right)=\phi_{x} G(\phi) \int_{x_{0}}^{x} d x^{\prime} F\left(x^{\prime}, \tau\right) .
$$

Once we define the Lagrangian

$$
L=\int_{-\infty}^{+\infty} d x \mathcal{L}
$$

the Euler-Lagrange evolution equations for the collective variables are obtained inserting a given Ansatz in this Lagrangian and using either expression (B6) or (B7). This procedure is the generalization of the Lagrangian formalism developed in [14].

Using the Rice Ansatz defined by (14) and (15) (for the SG and $\phi^{4}$ potentials, respectively) and after some straightforward calculations, we obtain the Lagrangian as a function of our collectives variables and their derivatives with respect to $\tau$

$$
\begin{aligned}
L\left(X, X^{\prime}, l, l^{\prime}\right)= & \frac{M_{0} l_{0}}{2 l}\left(X^{\prime}\right)^{2}+\frac{\alpha M_{0} l_{0}}{2 l}\left(l^{\prime}\right)^{2} \\
& -\frac{1}{2} M_{0} C^{2}(\tau)\left(\frac{l}{l_{0}}+\frac{l_{0}}{A^{2}(\tau) l}\right) \\
& -C^{2}(\tau) \int_{-\infty}^{+\infty} d x M\left(x, \tau, \phi, \phi_{x}\right) .
\end{aligned}
$$

Replacing $L\left(X, X^{\prime}, l, l^{\prime}\right)$ into the Euler-Lagrange equations,

$$
\frac{d}{d \tau}\left(\frac{\partial L}{\partial X^{\prime}}\right)=\frac{\partial L}{\partial X}
$$

$$
\frac{d}{d \tau}\left(\frac{\partial L}{\partial l^{\prime}}\right)=\frac{\partial L}{\partial l},
$$

and rewriting the equations of motion in the time variable $t$, we get

$$
\begin{gathered}
\dot{X}=\frac{a(t) P(t) l(t)}{M_{0} l_{0}}, \\
\frac{d P}{d t}=-(\beta+2 H) P-\frac{1}{a(t)}\left\{\int_{-\infty}^{+\infty} d \theta z(X+\theta l, t, \phi) \phi_{\theta}\right\}, \\
\alpha\left[\dot{l}^{2}-2(\beta+H) l i-2 \ddot{l}\right]=\frac{l^{2}}{l_{0}^{2}}\left(1+a^{2}(t) \frac{P^{2}}{M_{0}^{2}}\right)-\frac{1}{a^{2}} \\
+\frac{2 l^{2}}{M_{0} l_{0}} \int_{-\infty}^{+\infty} d \theta z(X+\theta l, t, \phi) \theta \phi_{\theta},
\end{gathered}
$$

where $\theta=[x-X(t)] / l$ and the dots denote the derivative with respect to $t$. These are the evolution equations for the collec- 
tive variables obtained for either of the two previous expressions of $M\left(x, t, \phi, \phi_{x}\right)$ Eq. (B6) or Eq. (B7) (in their respective regime of validity). Taking in these equations $\beta=0$ and $z=0$, we obtain the Eqs. (19)-(21) derived in Sec. III.

\section{GTWA}

In order to apply the GTWA we rewrite Eq. (B1) as

$$
\dot{\phi}=\psi,
$$

$$
\dot{\psi}=\frac{\phi_{x x}}{a^{2}(t)}-\frac{d U}{d \phi}-[H(t)+\beta(t)] \psi+z(x, t, \phi) .
$$

The procedure to obtain the CCs equations with the GTWA consists of inserting our specific functional form for $\phi$, Eq. (14) for sine-Gordon or (15) for $\phi^{4}$ potential, into Eq. (B15), multiplying the first equation by $\partial \psi / \partial X$ and the second one by $\partial \phi / \partial X$, taking their difference and integrating over $x$, equating the result with zero; and repeating the same procedure with $\partial \psi / \partial l$ and $\partial \phi / \partial l$. This gives us the same ordinary differential equations for $X(t), P(t)$, and $l(t)$ as those obtained in the previous subsection [see Eqs. (B12)-(B14)].
[1] A. C. Scott, Nonlinear Science (Oxford University, Oxford, 1999).

[2] M. A. Collins, A. Blumen, J. F. Currie, and J. Ross, Phys. Rev. B 19, 3630 (1979); 19, 3645 (1979).

[3] Kalman B. Migler and Robert B. Meyer, Phys. Rev. Lett. 66, 1485 (1991).

[4] H. K. Ebraheem, J. L. Shohet, and A. C. Scott, Phys. Rev. Lett. 88, 235003 (2002).

[5] J. L. Shohet, B. R. Barmish, H. K. Ebraheen, and A. C. Scott, Phys. Plasmas 11, 3877 (2004).

[6] A. Linde, Particle Physics and Inflationary Cosmology (Harwood Academic Publishers, Chur, Switzerland, 1990).

[7] P. Anninos, S. Oliveira, and R. A. Matzner, Phys. Rev. D 44, 1147 (1991).

[8] D.-H. Han, J.-G. Zhu, J. H. Judy, and J. M. Sivertsenb, Appl. Phys. Lett. 70, 664 (1997); L. Lanotte, G. Ausanio, V. Iannotti, G. Pepe, G. Carotenuto, P. Netti, and L. Nicolous, Phys. Rev. B 63, 054438 (2001).

[9] D. J. Grove and D. M. Meade, Nucl. Fusion 25, 1167 (1985).

[10] S. Weinberg, Gravitation and Cosmology (Wiley, New York, 1972).

[11] M. J. Rice and E. J. Mele, Solid State Commun. 35, 487 (1980).

[12] G. M. Wysin, F. G. Mertens, A. R. Völkel, and A. R. Bishop, in Nonlinear Coherent Structures in Physics and Biology, edited by K. H. Spatschek and F. G. Mertens, NATO Advanced Studies Institute, Series B: Physics (Plenum, New York, 1994),
Vol. 329; F. G. Mertens, H. J. Schnitzer, and A. R. Bishop, Phys. Rev. B 56, 2510 (1997).

[13] N. R. Quintero, A. Sánchez, and F. G. Mertens, Phys. Rev. E 62, 5695 (2000).

[14] N. R. Quintero and E. Zamora-Sillero, Physica D 197, 63 (2004).

[15] Sara Cuenda and Angel Sánchez, Chaos 15, 023502 (2005).

[16] N. R. Quintero, A. Sánchez, and F. G. Mertens, Phys. Rev. Lett. 84, 871 (2000).

[17] David K. Campbell, Jonathan F. Shonfeld, and Charles A. Wingate, Physica D 9, 1 (1983).

[18] N. R. Quintero and Panayotis G. Kevrekidis, Phys. Rev. E 64, 056608 (2001).

[19] F. G. Bass, Yu. S. Kivshar, V. V. Konotop, and Yu. A. Sinitsyn, Phys. Rep. 157, 63 (1988); Yuri S. Kivshar and Boris A. Malomed, Rev. Mod. Phys. 61, 763 (1989).

[20] D. W. McLaughlin and A. C. Scott, Phys. Rev. A 18, 1652 (1978).

[21] A. Sánchez and A. R. Bishop, SIAM Rev. 40, 579 (1998).

[22] G. Costantini, F. Marchesoni, and M. Borromeo, Phys. Rev. E 65, 051103 (2002); M. Salerno and Y. Zolotaryuk, ibid. 65, 056603 (2002); L. Morales-Molina, N. R. Quintero, F. G. Mertens, and A. Sánchez, Phys. Rev. Lett. 91, 234102 (2003).

[23] The width and the center of the kink position for the PDE results are obtained fitting the Rice Ansatz, Eq. (14) or Eq. (15), to the kink profile given by the PDE evolution (6). 